\title{
Dual Plating Osteosynthesis Technique used for Fixation of Inter - Condylar Distal Humerus Fractures via Transolecranon Approach
}

\author{
Jagdesh Kumar, Muhammad Soughat Katto, Malik Wasim Ahmed, Muhammad Jamil, \\ Irfan Muhammad Rajput, Adeel Ahmed Siddiqui, Ghulam Mustafa Kiam Khani, \\ Mehtab Ahmed Pirwani
}

\section{ABSTRACT}

INTRODUCTION: The dual plate osteosynthesis technique for fixation of inter-condylar distal humerus fracture is now considered an accepted treatment modality. It provides a rigid fixation of fracture fragments to enable early post-operative mobilization for good outcome.

OBJECTIVE: The purpose of study is to assess the morbidity and clinico-radiological outcome of the dual plating osteosynthesis technique used for fixation of inter-condylar distal humerus fractures (DHF) via trans-olecranon approach

METHODOLOGY: This perspective cross sectional study was carried at Department of Orthopaedic Surgery, Dow Medical College / Civil Hospital Karachi from June 2014 to March 2016. A total of 21adult patients with comminuted inter-condylar fracture of the distal humerus operated with dual plating osteosynthesis technique via trans-olecranon approach were evaluated clinically and radiologically for functional outcome based on Jupiter criteria including alignment and fracture union after a mean follow-up of 8 months. Post surgical complications were noted. Fractures were classified according to Muller et al (AO). Patients with polytrauma, pathological fracture, open fracture and a fracture more than 2 week old were excluded from the study.

RESULTS: Out of the 21 patients, $38 \%$ were female and $62 \%$ were male. $52.3 \%$ had left elbow while $47.6 \%$ had right elbow involvement. The mean age was 31 years (range 20 - 50 years). Mean time between injury and internal fixation was 4.8 days. The mean follow-up period was 8 months. As per AO classification, 10 cases were $C 1,7$ cases $C 2$ while 4 cases were in $C 3$. The most common cause of fracture was road traffic accident in 13 cases. All fractures achieved anatomical restoration of articular surface and were united in average $\mathbf{1 4 . 6}$ weeks. The clinical results were evaluated for functional outcome based on Jupiter criteria. According to that criterion, satisfactory results were obtained in all patients. We had no instance of postoperative deep infection and neurological complications, only Backing of olecranon K-wires were noted in 5 patients $(23.8 \%)$.

CONCLUSIONS: We conclude that dual plate osteosynthesis technique is an effective procedure for fixation of inter-condylar distal humerus fracture, achieves rigid fixation and hence, early mobilization. Additionally use of olecranon osteotomy offers best fracture exposure of distal humerus. Complications were minimal and healing satisfactory.

KEYWORDS: Trans-olecranon approach, dual plate osteosynthesis, inter - condylar distal humerus fracture

This article may be cited as: Kumar J, Katto MS, Ahmed MW, Jamil M, Rajput IM, Siddiqui AA, et al. Dual Plating Osteosynthesis Technique used for Fixation of Inter - Condylar Distal Humerus Fractures via Transolecranon Approach. J Liaquat Uni Med Health Sci. 2017;16 (03):139-44. doi: 10.22442/jlumhs.171630522

\section{INTRODUCTION}

Inter-condylar distal humerus fractures (DHF) in adult is one of the most problematic fracture that requires not only accurate reduction of the articular surface and restoration of fracture anatomy of the distal humerus but also require a rigid fixation of the fracture fragments to enable early postoperative mobilization ${ }^{1-5}$
A number of techniques have been used to treat such inter-condylar DHF in adult; all of them, fixation with dual plating through posterior approach with an olecranon osteotomy is now considered an accepted treatment modality ${ }^{1-3,6-9}$. Recently several authors have pointed out the importance of surgical approach, rigid fixation and early elbow mobilization for good 
outcome $^{1-14}$. This method of fixation provides rigid fixation; while use of posterior approach with olecranon osteotomy minimize the risk of triceps injury thus allowing early post-operative rehabilitation.

The current study was carried out to assess the morbidity and clinico-radiological outcome of the dual plating osteosynthesis technique used for fixation of intercondylar DHF via transolecranon approach.

\section{MATERIAL AND METHODS}

This prospective, cross-sectional study was conducted at department of Orthopaedic Surgery, Dow Medical College / Civil Hospital, Karachi from June 2014 to March 2016. In this study a total of 21 consecutive patients operated via trans-olecranon approach for comminuted inter-condylar fracture of the distal humerus with dual plating osteosynthesis technique were enrolled for outcome assessment. Patients with polytrauma, pathological fracture, open fracture and a fracture more than 2 week old were excluded from the study.

Fractures were classified according to Muller et al (AO). Radiographs of injured elbow (anteroposterior and lateral view) were taken to evaluated fracture and CT Scan-3D of elbow (in the presence of metaphyseal and articular comminution).

\section{Sample technique}

Non-probability - purposive sampling technique was used to select 21 patients, who sustained inter-condylar fracture of the distal humerus requiring surgery.

\section{SAMPLE SIZE CALCULATION}

Determination of sample size was based on an epidemiological study in the bone and joint journal, where distal humeral fractures accounted for $2 \%$ of total adult fractures ${ }^{26,27}$. Supracondylar / intercondylar distal humerus fractures are even less common, having only a $0.3 \%-1 \%$ incidence ${ }^{26,27}$.

Using Fishers' formula

$\mathrm{n}=\mathrm{Z} 2 \mathrm{PQ} / \mathrm{D} 2$

Where

$\mathrm{n}$ is the estimated sample size.

$\mathrm{Z2}$ is the score of confidence interval at $95 \%$ and is 1.962.

$P$ is the prevalence in this case at $1 \%$ and $Q$ is $1-P$. D2 is the degree of error which is 0.052

Therefore

$\mathrm{n}=1.962 \times 0.01(1-0.01) / 0.052$

$\mathrm{n}=16$ patients

\section{DATA COLLECTION AND STATISTICAL ANALYSIS}

Data collected over a predesigned data collection sheets and analyzed using SPSS version 19 and presented in form of tables and graphs.

\section{Surgical procedure \& post-operative management}

After detailed counseling and before surgery a written consent was taken. Preoperative antibiotic prophylaxis was given, one hour before surgery. Surgery was done under general anesthesia with patient in the lateral position. After placement of upper arm pneumatic tourniquet and draping, a longitudinal dorsal incision was given with radial deviation to the olecranon tip in all the patients. The ulnar nerve was isolated and protected to prevent iatrogenic damage. Due to difficulty in reposition of osteotomy accurately, the proximal ulna was predrilled with two parallel 1.8 $\mathrm{mm}$ K-wire. The transverse olecranon osteotomy was made by using a thin oscillating saw about 2 centimeters distal to the olecranon tip. The olecranon fragment along with the triceps muscle was elevated off the posterior surface of humerus to expose the fracture fragments.

The first step was to reduce and fix the articular surface and both medial and lateral condyles together with a partially threaded cancellous screw. The $2^{\text {nd }}$ step was to anatomically reduce and fix the condyles to the humeral shaft with bicolumnar/medial and lateral plates to ensure rigid and stable fixation. On completion of humeral fixation, the olecranon was fixed back with two parallel K-wires along with tension band wire. The parallel K-wires were passed through same predrilled hole. The tourniquet was deflated and homeostasis achieved. Wound was properly closed by layers over a negative suction drain. Postoperatively, backslab was given only when the bone quality was poor and fixation of fracture was questionable.

\section{Rehabilitation protocol and outcome assessment:}

All patients had received intravenous broad spectrum antibiotics for 2 days, followed by oral antibiotic for further 5 days to prevent infection. The patients were discharges on the $3^{\text {rd }}$ to $4^{\text {th }}$ post-op day and ask to return on day 14 for removal of stitches. The passive and active assisted elbow range of motion exercises as pain permitted was started in patients with good bone quality and rigid fixation usually on the $2^{\text {nd }}$ or $3^{\text {rd }}$ postoperative day. The patients were allowed to carry weight only after radiological evidence of union. Patients were followed every 4 weeks interval. At each visit, patients were evaluated clinically for functional outcome based on Jupiter criteria ${ }^{16}$ and radiologically for reduction, alignment and fracture union. Union was defined as the presence of bridging callus or the disappearance of the fracture line on three of four cortices seen on two radiographic views. Post surgical complications were also noted (ie, wound infection, nerve palsy, nonunion of fractures and osteotomy, prominent K-wires and implant failure). 


\section{RESULTS}

Out of the 21 analyzed patients, $8(38 \%)$ were female and $13(62 \%)$ were male. $11(52.3 \%)$ had left elbow while $10(47.6 \%)$ had right elbow involvement. Average age was 31 years (Range 20 - 50 years). Mean time between injury and internal fixation was 4.8 days (Range 0-14 days), which was mostly due to delay in reporting to the hospital. The mean follow-up period was 8 months (Range 6 to 13 months). As per AO classification, 10 cases were $C 1,7$ cases $C 2$ while 4 cases were in $\mathrm{C} 3$. The most common mode of fracture was traffic accident in 13, followed by fall in 7 and direct trauma in 1.

Out of 21 bicolumnar fracture, 8 fractures were fixed by $1 / 3$ semitubular plate medially and small fragment dynamic compression plate on the lateral side while 13 fractures were fixed by precontoured anatomical locking plates. We had not done a comparison, but in term of fixation, precontoured anatomical LCP is found as an implant of choice especially in humerus with decreased bone mineral quality or in the presence of a metaphyseal comminution. However, in term of costs, precontoured anatomical LCP was found to be over seven times more expensive than the conventional plates.

All fractures achieved anatomical restoration of articular surface in the early postoperative radiograph and were united in average 14.6 weeks (Range 10-28 weeks). Non-union of humerus was not noted. The clinical results were evaluated for functional outcome based on Jupiter criteria ${ }^{9}$ (Table No. 1). These criteria consist of range of elbow motion (ROM) and subjective findings.

According to the range of motion, the results were labeled to be excellent in $12(57 \%)$, good in $7(33 \%)$ and fair in $2(9.5 \%)$.When the results were graded according to the subjective findings, the results were labeled to be excellent in $15(71.4 \%)$, good in 5 $(23.8 \%)$ and fair in $1(4.7 \%)$.

We had no instance of postoperative deep infection

TABLE I: FUNCTIONAL SCORING SYSTEM, JUPITER CRITERIA

\begin{tabular}{|l|c|l|l|}
\hline \multicolumn{2}{|c|}{ Range of motion (degrees) } & \multicolumn{2}{l|}{ Subjective evaluation } \\
\hline $\begin{array}{c}\text { Loss of } \\
\text { Extension }\end{array}$ & \multicolumn{1}{|c|}{ Flexion } & \multicolumn{1}{|c|}{ Pain } & Disability \\
\hline Excellent & $<15 \quad 130$ & None & None \\
\hline Good & $<30120$ & Occasional & Minimum \\
\hline Fair & $<40 \quad 90-120$ & With activity & Moderate \\
\hline Poor & $<40<90$ & Variable & Severe \\
\hline
\end{tabular}

and neurovascular complications. All olecranon osteotomies were united without complications, only backing of olecranon K-wires were noted in 5 patients $(23.8 \%)$, but this did not affect the elbow range of motion.

\section{Pre-operative x-ray: A- AP View, B- Lateral view}

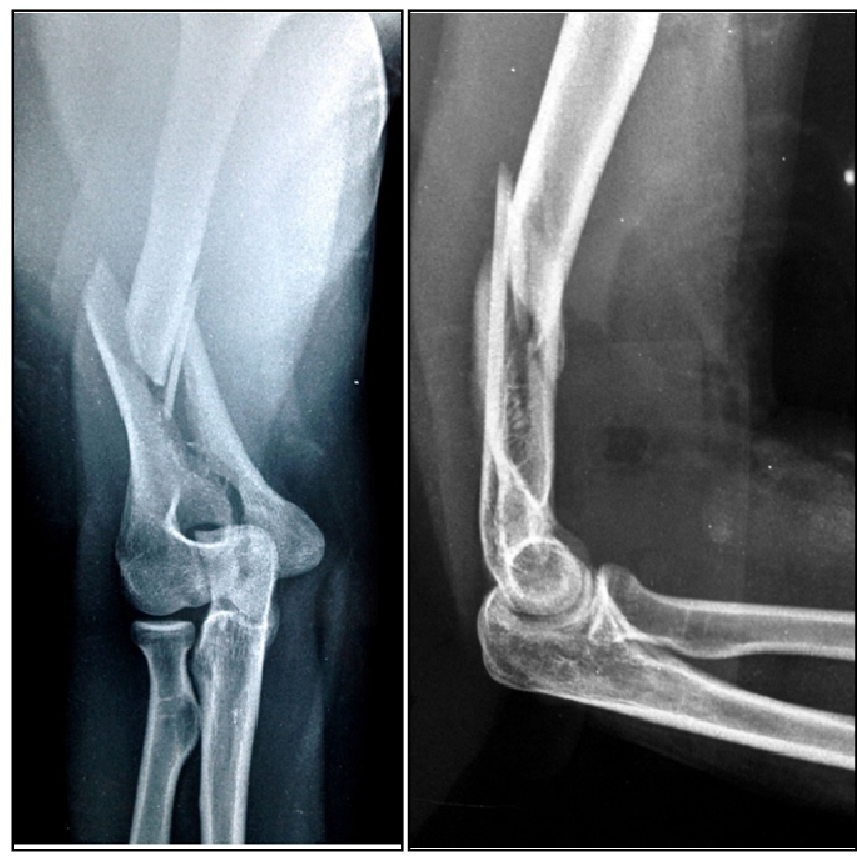

Post-operative x-ray: Anteroposterior and Lateral view showing fixation with $1 / 3^{\text {rd }}$ semitubular plate medially and small fragment dynamic compression plate on the lateral side

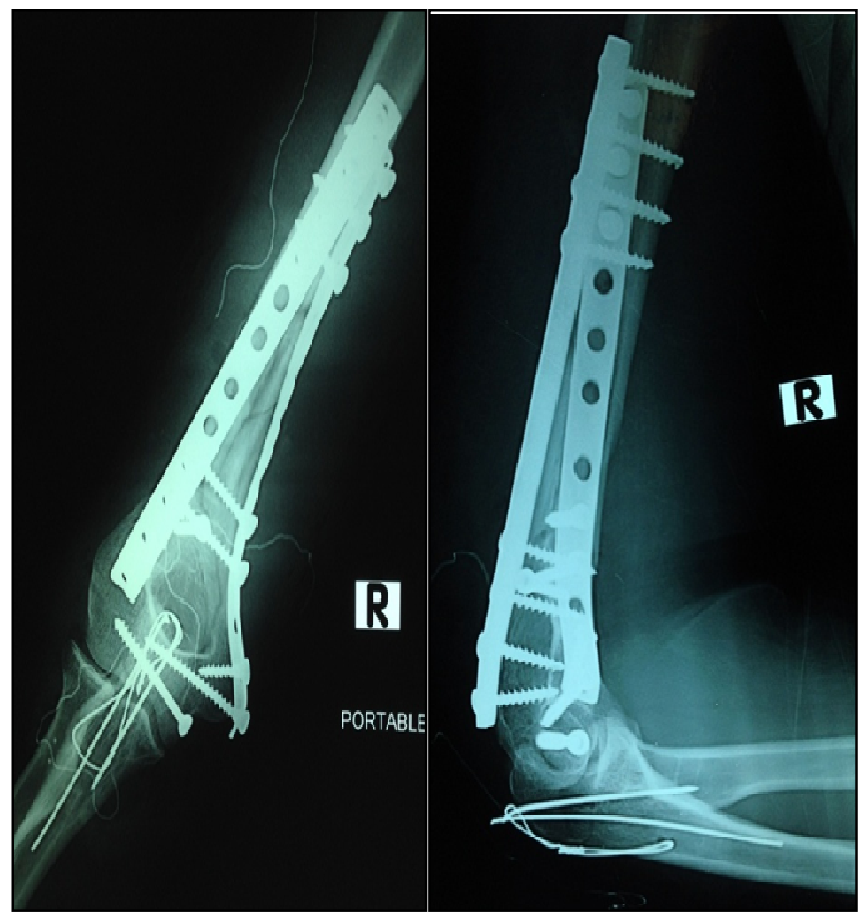




\section{Intra-operative x-ray showing Intercondylar distal humerus fracture \\ Intra-operative x-ray after fixation with dual plate osteosynthesis}

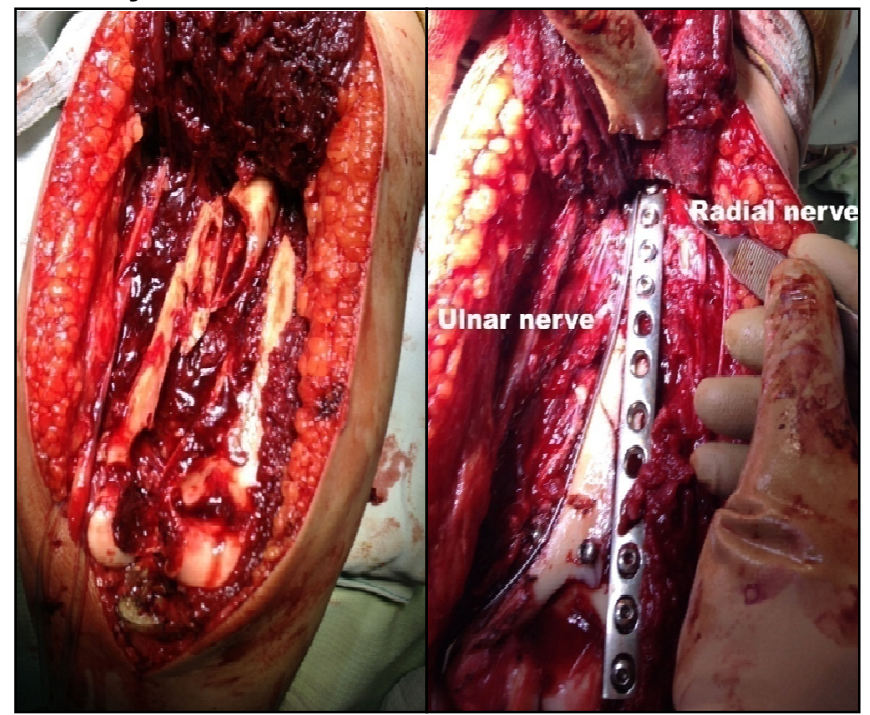

Post-operative x-ray after 9 month follow-up
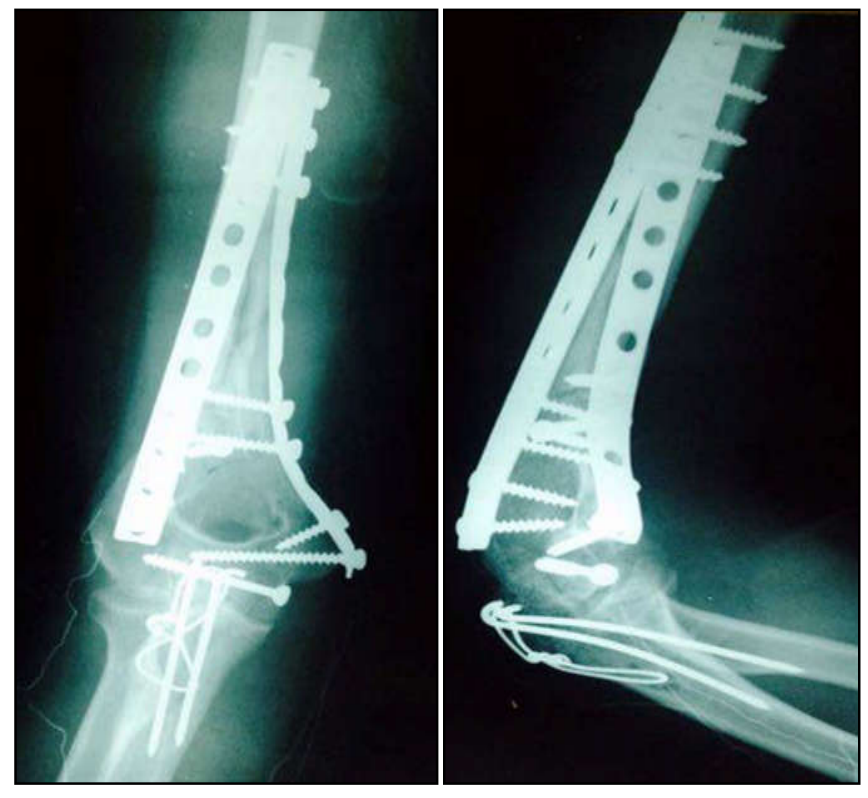

\section{DISCUSSION}

Although intraarticular distal humerus fractures are rare, they present a clinical challenge for orthopaedic surgeons due to the complex anatomy of the distal humerus and difficulty in exposing the fracture. The main surgical goal of fixation of such fractures is to achieve a stable joint with a good range of pain free movement ${ }^{1-5}$. Many fixation methods have been proposed; the use of K-wires, screw fixations, Y-shaped plates, single plates and double plates. Among them the dual plating osteosynthesis through a posterior approach with an olecranon osteotomy is now considered an acceptable treatment modality ${ }^{1-3,6-}$

${ }^{9}$, It offers excellent posterior visualization of the distal humerus, without any untoward effect on triceps strength and postoperative rehabilitation ${ }^{6-14}$. Recently many national and international studies have focused attention on the importance of surgical approach and stable fixation for early elbow mobilization ${ }^{1-14,18,19}$.

In this study we had used a posterior approach with an olecranon osteotomy in all cases. At final follow-up, in this study according to the Jupiter criteria $90 \%$ patient had excellent to good functional outcome that are comparable to other's result using same technique and approach. Jupiter and colleagues ${ }^{16}$ using same approach reported $79 \%$ good to excellent outcome in series of 34 patients. Swamy A et al reported $76.6 \%$ excellent to good result in series of 30 patients ${ }^{17}$. Mang I et al reported $80 \%$ excellent to good result in series of 25 patients ${ }^{18}$. Khalid $\mathrm{MU}$ et al using either trans-olecranon or triceps sparing approach reported $88.2 \% \%$ "excellent" to "good" results ${ }^{19}$ while $\mathrm{Li} \mathrm{SH}$ et al reported $84 \%$ excellent to good functional outcome after a mean follow-up of 30 months in AO type C distal humerus fractures using olecranon osteotomy ${ }^{2}$.

The complications encountered in the operative treatment of distal humeral fractures as reported by various authors ${ }^{20-25}$ includes: wound infection, nerve injuries, nonunion of fractures and osteotomy, prominent K-wires, heterotopic ossification, stiffness, pain and implant failure. However in current study, we had no instance of postoperative deep infection. Superficial wound infection was noted in $6(16.6 \%)$ patients and was successfully resolved with dressings and change of oral antibiotics. Incidence of ulnar nerve injury has been reported in $5-15 \%$ of patients ${ }^{20-}$ ${ }^{21}$, in this study, we had no incidence of iatrogenic ulnar nerve palsy. This may be due to direct identification and mobilization of ulnar nerve during surgery.

The most frequently quoted complications associated with olecranon osteotomy are non-union and backing of olecranon K-wires ${ }^{22-25}$, However in this study no case of olecranon nonunion was encountered while delayed union was noted in 3 cases and symptomatic prominent K-wires were noted in 5 cases. All fractures as well as the olecranon osteotomy healed within the expected time; there was no instance of fixation failure. Presumably this may be due to rigid dual plate osteosynthesis and satisfactory anatomical fracture reduction.

In this study, $10(47.6 \%)$ patients regained full range of motion within 12 - 16 weeks whereas $6(28.5 \%)$ patients had restriction of elbow motion of $10-20$ degrees, $3(14 \%)$ patients had restriction of around 20 - 30 degrees and 2(9.5\%) patient had restriction of 
more than 40 degree. Patients with restriction in movement had severe metaphyseal and articular comminution compared with others.

In this study, we had used both conventional plates and precontoured anatomical locking compression plates (LCP). We had not done a comparison, but in term of fixation, precontoured anatomical LCP is found as an implant of choice especially in humerus with decreased bone mineral quality or in the presence of a metaphyseal comminution. However, in term of costs, precontoured anatomical LCP was found to be over three times more expensive than the conventional plates.

\section{CONCLUSION}

We conclude that dual plating osteosynthesis technique is an effective procedure for fixation of inter-condylar distal humerus fractures. It is a safe procedure to achieve rigid fixation and hence, early mobilization. Additionally use of olecranon osteotomy offers best fracture exposure for articular reconstruction, allowing bicolumnar plating while monitoring the ulnar nerve. Complications were minimal and healing satisfactory.

\section{REFERENCES}

1. Puchwein $P$, Wildburger $R$, Archan $S$, Guschl M, Tanzer K, Gumpert R. Outcome of type C (AO) distal humeral fractures:follow-up of 22 patients with bicolumnar plating osteosynthesis. J Shoulder Elbow Surg.2011; 20(4):631-6.

2. Li SH, Li ZH, Cai ZD, Zhu YC, Shi YZ, Liou J, et al. Bilateral plate fixation for type $\mathrm{C}$ distal humerus fractures: experience at a single institution. Int Orthop. 2011; 35(3):433-8.

3. Liu YK, Xu H, Liu F, Wang YH, Tao R, Cao Y, et al. Treatment of type $C$ intercondylar fractures of distal humerus using dual plating. Zhonghua Wai Ke Za Zhi. 2009; 47:892-5.

4. Sarkhel S, Bhattacharyya S, Mukherjee S. Condylar orientation plating in comminuted intraarticular fractures of adult distal humerus. Indian J Orthop. 2015;49(5): 523-8.

5. Patel J, Motwani G, Shah H, Daveshwar R. Outcome after internal fixation of intraarticular distal humerus ( $A O$ type $B$ \& $C$ ) fractures: Preliminary results with anatomical distal humerus LCP system. J Clin Orthop Trauma. 2017; 8(1):63-7.

6. Jung SW, Kang SH, Jeong M, Lim HS.Triangular Fixation Technique for Bicolumn Restoration in Treatment of Distal Humerus Intercondylar Fracture. Clin Orthop Surg. 2016; 8(1):9-18.

7. Dasheng Tian, Juehua Jing, Jun Qian, Jianming Li. Comparison of two different double-plate fixation methods with olecranon osteotomy for intercondylar fractures of the distal humeri of young adults. Exp Ther Med. 2013;6(1):147-151.

8. Doornberg JN, van Duijn PJ, Linzel $D$, et al: Surgical treatment of intra-articular fractures of the distal part of the humerus. Functional outcome after twelve to thirty years. J Bone Joint Surg Am. 2007; 89(7):1524-32.

9. Dreyfuss D, Eidelman M. Treatment of complex intercondylar humeral fractures in adolescents by open reduction and internal fixation through the transolecranon approach. J Pediatr Orthop B. 2014; 23(4):364-8.

10. Elmadag M, Erdil M, Bilsel K, Acar MA, Tuncer N, Tuncay I. The olecranon osteotomy provides better outcome than the triceps lifting approach for the treatment of distal humerus fractures. Eur $\mathrm{J}$ Orthop Surg Traumatol. 2014; 24(1):43-50.

11. Khan KM, Kumar $S$, Minhas MS, Bhatti A. Functional outcome following olecranon osteotomy approach for fixation of $T / Y$ fractures of distal humerus. J Pak Med Assoc. 2015; 65(11 suppl 3):S25-7.

12. Mardanpour K, Rahbar M. Open reduction and internal fixation of intraarticular fractures of the humerus: evaluation of 33 cases. Trauma Mon. 2013; 17(4): 396-400.

13. Gupta RK, Gupta V, Marak DR. Locking plates in distal humerus fractures: study of 43 patients. Chin J Traumatol. 2013;16(4):207-11.

14. Gupta V, Kalsotra N, Gupta R, Motten T, Singh M, Kamal $Y$, et al. Operative management of intercondylar fractures of the distal end humerus in adults. The Internet Journal of Orthopedic Surgery $2009 ; 17: 1-5$.

15. Müller ME, Nazarian S, Koch P,Schatzker J. The Comprehensive Classification of Fractures of Long Bones. 1st ed. Berlin, Germany: Springer, 1990.

16. Jupiter JB, Neff $U$, Holzach $P$, Allgower $M$. Intercondylar fractures of the humerus. An operative approach. J Bone Joint Surg. 1985;67:226-39.

17. Swamy A. Thirty cases of distal humerus intra-articular fractures treated by open reduction and internal fixation: A 3-year review. Med J DY Patil Univ. 2012;5:114-7.

18. Mang I, Taufiq I, Najjad MKR, et al. Functional Outcome of Elbow Reconstruction after Using Precontoured Locking Compression Plate. J Pak Orthop Assoc. 2014; 26(1):35-8.

19. Khalid MU, Saeed KM, Akhter M. A comparison of functional outcome of intercondylar fracture of distal humerus managed by olecranon osteotomy approach versus triceps sparing approach in 
adults. J Pak Med Assoc. 2015; 65(11 Suppl 3):S119-22.

20. Kundel K, Braun W, Wieberneit J, Ruter A. Intraarticular distal humerus fractures. Factors affecting functional outcome. Clin Orthop Relat Res. 1996; 332:200-8.

21. Gupta $R$, Khanchandani $P$. Intercondylar fractures of the distal humerus in adults: a critical analysis of 55 cases. Injury. 2002; 33(6):511-5.

22. Macko D, Szabo RM. Complications of tension band wiring of olecranon fractures. J Bone Joint Surg Am.1985;67(9):1396-401.

23. Horne JG, Tanzer TL. Olecranon fractures: A review of 100 cases. J Trauma. 1981; 21:469-72.
24. Ring D, Gulotta L, Chin K, Jupiter JB. Olecranon osteotomy for exposure of fractures and nonunions of the distal humerus. $J$ Orthop Trauma. 2004; 18(7):446-9.

25. Gainor BJ, Moussa F, Schott T. Healing rate of transverse osteotomies of the olecranon used in reconstruction of distal humerus fractures. J South Orthop Assoc. 1995;4:263-8.

26. Court-Brown CM, Caesar B. Epidemiology of adult fractures: a review.Injury.2006; 37(8):691-7.

27. Robinson CM, Hill RM, Jacobs N, Dall G, Court-Brown CM. Adult distal humeral metaphyseal fractures: epidemiology and results of treatment. J Orthop Trauma. 2003; 17(1):38-47.

AUTHOR AFFILIATION:

Dr. Jagdesh Kumar (Corresponding Author)

Assistant Professor

D.U.H.S \& Civil Hospital Karachi, Sindh-Pakistan.

Email: Jagdesh@doctor.com

Dr. Muhammad Soughat Katto

Assistant Professor

D.U.H.S \& Civil Hospital Karachi, Sindh-Pakistan.

Dr. Malik Wasim Ahmed

Assistant Professor

D.U.H.S \& Civil Hospital Karachi, Sindh-Pakistan.

Dr. Muhammad Jamil

Assistant Professor

D.U.H.S \& Civil Hospital Karachi, Sindh-Pakistan.

Dr. Irfan Muhammad Rajput

D.U.H.S \& Civil Hospital Karachi, Sindh-Pakistan.

Dr. Adeel Ahmed Siddiqui

D.U.H.S \& Civil Hospital Karachi, Sindh-Pakistan.

Prof. Ghulam Mustafa Kaimkhani

D.U.H.S \& Civil Hospital Karachi, Sindh-Pakistan.

Dr. Mehtab Ahmed Pirwani

Assistant Professor

D.U.H.S \& Civil Hospital Karachi, Sindh-Pakistan. 